\title{
Neoliberalización del territorio y movilidad urbana, una agenda de investigación
}

Territorial Neoliberalization and Urban Mobility. Towards a Research Agenda

Neoliberalização do território e mobilidade urbana, uma agenda de pesquisa

Fernando Calonge Reillo*

Recibido: 7 de julio de 2014

Aprobado: 29 de octubre de 2014

Doi: dx.doi.org/10.12804/territ32.2015.07

Para citar este artículo:

Calonge, R. F. (2015). Neoliberalización del territorio y movilidad urbana, una agenda de investigación. Territorios, 32, 133-156. Doi: dx.doi.org/10.12804/territ32.2015.07

\begin{abstract}
* Doctor en Sociología por la Universidad Complutense de Madrid. Coordinador de la Maestría y el Doctorado en Movilidad Urbana, Transporte y Territorio, Centro Universitario de Tonalá, Universidad de Guadalajara, México.Correo electrónico: fercalonge@yahoo.es Dirección: C/ Rio Lerma 501, Col. Loma Bonita Ejidal, CP. 45608, Tlaquepaque, Jalisco, México.
\end{abstract}


Palabras clave

Movilidad urbana, neoliberalismo,

neoliberalización del territorio, desinversiones, especulación, polaridades urbanas.

Keywords

Urban mobility, neoliberalism, territorial neoliberalization, disinvestmens, speculation, urban polarities.

Palavras-chave

Mobilidade urbana, neoliberalismo, neoliberalização do território, desinvestimento, especulação, polaridades urbanas.

territarias 32

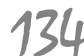

\section{RESUMEN}

El presente artículo ubica los estudios de la movilidad urbana dentro del marco más general de análisis del neoliberalismo presente. Dado que los discursos sobre el neoliberalismo en ocasiones son demasiado abstractos y generales, se opta por ofrecer una versión teórica de rango medio que aproxime ambos tipos de fenómenos. Así se examinan distintos procesos de neoliberalización del territorio, para comprender de qué forma inciden en las movilidades urbanas y para comprender cómo estas movilidades sirven para reproducir y ampliar aquella neoliberalización. El objetivo último es establecer campos temáticos e hipótesis que puedan tomar futuros estudios en la construcción de una agenda de investigación sobre movilidad urbana y neoliberalismo.

\section{ABSTRACT}

This paper tries to situate urban mobility studies into the more general framework of present neoliberalism. Since neoliberalism discourses quite often are too general and abstract, we transform them into a middle range theory in order to make closer both phenomena. In so doing we explore different process of territorial neoliberalization to understand how they influence urban mobilities, and to discover how urban mobilities reproduce and extend such neoliberalization. The main purpose is to establish hypothesis and topics for succeeding studies in constructing a research agenda on urban mobility and neoliberalism.

\section{RESUMO}

O presente artigo localiza os estudos da mobilidade urbana dentro do marco mais geral de análise do neoliberalismo presente. Dado que os discursos sobre o neoliberalismo em ocasiões são demasiado abstratos e gerais, opta-se por oferecer uma versão teórica de categoria meia que aproxime ambos os tipos de fenómenos. Assim examinam-se distintos processos de neoliberalização do território para compreender de que forma incidem nas mobilidades urbanas e para compreender como estas mobilidades servem para reproduzir e ampliar aquela neoliberalização. O objetivo último é estabelecer campos temáticos e hipótese que possam tomar futuros estudos na construção de uma agenda de pesquisa sobre mobilidade urbana e neoliberalismo. 


\section{Introducción}

Hasta el presente, las aportaciones de la economía, la sociología y la geografía han contribuido a conformar un corpus teórico muy dilatado sobre la naturaleza del neoliberalismo. En los últimos veinte años se ha construido un discurso más o menos coherente, de perfiles, a veces poco definidos, sobre la emergencia de un sistema de libre mercado a escala mundial, sus repercusiones de desarrollo desigual, de polarización social o de fragmentación de los territorios y las urbes. Al mismo tiempo, los estudios del transporte y la movilidad urbana han proseguido, atendiendo a fenómenos como la reestructuración de los servicios públicos de transporte, el aumento exponencial de los automóviles en las ciudades o los problemas cada vez menos aplazables de la sustentabilidad social y ambiental.

No obstante, aunque se observa una clara relación entre ambos órdenes de discursos, el del neoliberalismo y los propios de los estudios de la movilidad urbana, faltan trabajos que se encarguen explícitamente de expresar las formas concretas que adquiere dicha relación. El documento que aquí se presenta intenta contribuir a superar esta deficiencia al establecer una agenda de investigación, lo más detallada posible, que indique de qué maneras los procesos de reorganización del capitalismo global inciden en los condicionantes que enmarcan la movilidad en las ciudades presentes. Dado que los discursos sobre el neoliberalismo son de orden macro y totalizador -incluyendo abstractas construcciones teóricas sobre el desarrollo de la economía mundial, carentes en ocasiones de contenido empírico concreto- se ha decidido seguir la recomendación de Robert K. Merton (2007) de buscar órdenes de fenómenos y teorías meso que sirvan para aterrizarlos y reconducirlos hacia la referida agenda de investigación. Dicha reconducción se realizará sobre los procesos más particulares de la neoliberalización del territorio.

Para materializar estas intenciones, en un primer momento se ofrecerá un breve recuento de los principales rasgos del neoliberalismo, los fundamentales, para tener un entendimiento cabal de la discusión que seguirá. A continuación, se introduce una discusión sobre cómo el territorio se transforma, bajo el capitalismo neoliberal, en un circuito crucial para estabilizar y también espolear la acumulación capitalista. Después de examinar cómo este régimen capitalista transforma el territorio urbano, se realizará un recuento de las diferentes formas en las que se modifican las condiciones para las movilidades urbanas ${ }^{1}$ y la redefinición de los sistemas de transporte. Para ello, se revisa la incidencia para la movilidad urbana de fenómenos como las reestructuraciones urbanas, la especulación sobre el territorio, o la constitución de profundas polaridades socio-espaciales.

\section{Una breve caracterización del neoliberalismo}

Es obvio señalar que todas las aportaciones que se han realizado sobre el neoliberalismo no pueden quedar resumidas con
${ }^{1}$ Según Elliot y Urry (2010: 15-16) las movilidades están compuestas por movimientos físicos de personas, objetos y mercancías y por movimientos imaginarios $y$ virtuales de comunicaciones o información. En este estudio restringiremos el contenido de movilidad urbana a los movimientos físicos de personas y objetos que se producen por medio(y no solo al interior) de losentornos urbanos. territarias 32

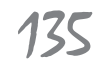


justicia en un texto como el presente. Sin embargo, se intentará ofrecer sus principales rasgos para poder derivar luego un entendimiento cabal de cómo se expresa bajo la forma de la neoliberalización del territorio.

El pilar sobre el que se asientan los discursos neoliberales consiste en el axioma de que los mercados abiertos, competitivos y sin regulación suponen el mecanismo más eficiente para el desarrollo socioeconómico (Peck, Theodore \& Brenner, 2009). Desde un período antecedente donde las doctrinas keynesianas aconsejaban la intervención del estado en la dirección de la economía para apaciguar las recurrentes crisis del capitalismo (Swyngedouw, 2004), el neoliberalismo supuso un retroceso de la mayor parte de las políticas intervencionistas del estado. Esta transformación cobró forma por medio de la desregulación del estado a la actividad industrial y la bajada de sus impuestos, la privatización de las empresas públicas, el desmantelamiento de las formas colectivas de negociación laboral, el repliegue de las políticas de bienestar y de solidaridad social entre las diferentes capas de la población, el impulso a la movilidad y a las inversiones internacionales de capital, o por medio del refuerzo a las políticas de competencia interlocales de las diferentes regiones (Peck, Theodore \& Brenner, 2009).

Esta serie de políticas se materializó de forma muy diferente en función de los contextos sociopolíticos, institucionales y territoriales de cada uno de los países. De hecho, el neoliberalismo no habría que entenderlo tanto como un estado sino como varios procesos que abren vías de reconversión económica en función de la estructura institucional pasada y de la particularidad de los nuevos realineamientos económicos (Peck, Theodore \& Brenner, 2009).

Es este seguimiento a los procesos concretos de neoliberalización lo que evidencia que su naturaleza no suponga tanto una desaparición de la actividad del estado, sino su realineamiento en el sentido de construir, reforzar y generalizar las condiciones que garantizan el libre mercado (Brenner \& Theodore, 2005). De esta forma, el neoliberalismo habría que considerarlo no tanto como la materialización del viejo sueño liberal, consistente en el equilibrio logrado por la libre concurrencia de los actores individuales, sino como un sistema regulatorio donde el estado sigue manteniendo una fuerte intervención, solo que en el sentido de imponer relaciones sociales mercantilizadas (Brenner, Peck \& Theodore, 2010), gracias a la construcción de un nuevo marco institucional (Harvey, 2006).

Así, el neoliberalismo puede descomponerse en dos movimientos íntimamente relacionados. Uno de repliegue y otro de despliegue. En el primer movimiento, que se escenificó sobre todo en la década del ochenta y que tuvo sus adalides en los gobiernos de Ronald Reagan en Estados Unidos y de Margaret Thatcher en el Reino Unido, la labor fundamental fue el desmantelamiento de las instituciones estatales que habían caracterizado el período fordistakeynesiano antecedente bajo la creencia de que su supresión comportaría en automático el referido equilibrio de las relaciones 
mercantiles al interior de las sociedades civiles. Sin embargo, ante la evidencia de que esos movimientos de desregulación acarreaban una vuelta a las crisis en los procesos de acumulación, se procedió a desplegar todo un andamiaje institucional que entronizara el libre mercado, al tiempo que apaciguara algunos de los desajustes precedentes (Peck, Theodore \& Brenner, 2009). En este período de expansión se establecieron medidas para facilitar la reincorporación de las poblaciones marginadas a los mercados laborales o se fortalecieron los consorcios público-privados para la ampliación de las potencialidades de las regiones metropolitanas, siempre bajo el supuesto de garantizar las condiciones para la acumulación privada.

Ahora bien, existe una serie de fenómenos que diferencian el período neoliberal presente del propiamente liberal del siglo XIX. En primer lugar, hay que señalar el carácter radicalmente internacional de esta segunda oleada de liberalización. Esto en parte se debe a que ha cambiado el mercado por medio del cual se produce la referida liberalización. Mientras que en el siglo XIX, la liberalización se apoyó en la internacionalización de la extracción de materias primas y de la producción industrial, al presente la internacionalización se estará apoyando en los mercados financieros (French, Leyshon \& Wainwright). El tipo de intercambios que están componiendo las actuales interacciones económicas internacionales ya no se apoya tanto en bienes y productos, sino en el propio dinero. Así, los procesos de acumulación capitalistas no se producen mediante la extracción y producción de recursos, sino mediante los propios intercambios de capital, o de la supeditación al capital de nuevos espacios sociales como la seguridad o las solidaridades sociales. El que sean los mercados de capitales, y no los de bienes y productos los que soportan la internacionalización de la economía, hace que el proceso pueda cobrar mucha más intensidad e instantaneidad al perder su vinculación con las pesadas geografías de las minas, las plantaciones o las factorías del precedente capitalismo industrial.

Pero al mismo tiempo que sucede esta intensificación de la dimensión internacional del capitalismo, apoyada en los aspectos puramente financieros de las actividades económicas, es propio de esta fase presente de neoliberalismo el que se exprese por medio de una muy acusada heterogeneidad geográfica. Frente a los modelos del desarrollo del período keynesiano, que visualizaban una derrama homogénea del crecimiento económico a lo ancho de todos los territorios, bajo el neoliberalismo se aprecia que las mejoras en las comunicaciones y transportes redundan en una más fina especialización y subespecialización de los territorios, deparando otras tantas posibilidades de acumulación capitalista. Es lo que Harvey conoció como el desarrollo geográfico desigual, donde la acumulación se produce por la explotación de los agudos contrastes de desarrollo económico a lo ancho del planeta (Harvey, 2005). De este modo, la geografía contemporánea estaría marcada por diferenciales muy acusados de territarias 32

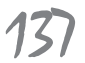


2 Es la estrategia que el propio Hackworth (2007) ha reconocido como excluir para expandir.

territarias 32 desarrollo económico, político y social que permitirían cristalizar nuevas oportunidades de inversión y acumulación capitalista (Soja, 1990).

\section{La neoliberalización del territorio como oportunidad para la formación del capital}

Según David Harvey (2008), la urbanización desempeña un papel muy importante dentro de los procesos de estabilización del capitalismo. Según su análisis, las condiciones de competencia extrema y de superproducción complican de sobremanera la acumulación dentro del circuito primario del capital (aquel que integra los procesos productivos), bajo la clásica consigna de Marx (2009) sobre el rendimiento decreciente de los beneficios. A este respecto, es funcional para el proceso de acumulación la constitución de un circuito secundario donde el capital pueda temporalmente absorberse para evitar esos desajustes y excedentes de producción dentro del circuito primario. Esencialmente, este circuito secundario, a juicio de Harvey (2008), lo constituye el mercado inmobiliario. En él pueden absorberse temporalmente los grandes excedentes del capital, de manera que no espoleen los tradicionales ciclos de superproducción y caída de rendimientos dentro del circuito primario. El segundo circuito del capital se constituye como un espacio estabilizador y que en sí mismo puede poner en marcha nuevos procesos de acumulación por medio de la no productiva especulación inmobiliaria (Harvey, 2008).
De esta forma, el territorio, fundamentalmente urbano, se convierte en uno de los instrumentos que mayor importancia $\mathrm{co}^{-}$ bran para la estabilidad de la acumulación del capital en el período neoliberal.

Bajo esta forma del capitalismo, una serie de reformas - como la supresión de trabas al movimiento de capitales, un tipo de gobernanza empresarial de las ciudades, o las certificaciones que las calificadoras internacionales realizan sobre las economías urbanas - ha facilitado que se produzca un movimiento sin límites del capital entre las distintas urbes, conformando una auténtica globalización del mercado inmobiliario (Mitchell \& Beckett, 2008). Así, esos efectos de estabilización, por medio de dicho mercado, presentan una cobertura global, como ha quedado demostrado, a contrario$r i$, por las repercusiones financieras globales que precipitó la crisis de las hipotecas en Estados Unidos en 2008.

Aquí, se advierte la nueva relación que adquieren los estados con el sostenimiento de estos procesos de apropiación capitalista de origen global. En ocasión de estas inversiones globales en el circuito secundario del capital, el estado trabaja por garantizar su rendimiento posterior, recurriendo a la tradicional estrategia de la zonificación que preserva el establecimiento de poblaciones y funciones no deseadas a su alrededor (Hackworth, 2007), o la más nueva gestión y fortificación de las fronteras urbanas para contenerlas. ${ }^{2}$ El hecho de que las ciudades compitan por captar los flujos de capital internacionales, asegurándoles sus rendimientos, explica que encaren la gestión 
cotidiana del territorio desde las políticas excluyentes de poblaciones no deseadas, antes que desde políticas de integración y cohesión sociales (Ortiz Gómez \& Zetter, 2004).

El que los intercambios y las inversiones alcancen la dimensión global en el presente no quiere decir que puedan prescindir de localizarse y territorializarse. Aunque sea por cada vez más breves períodos, los agentes económicos han de espacializar sus inversiones para sostener la recogida lo más ampliada posible de beneficios. Como ha quedado ya señalado, el capitalismo necesita localizarse en determinados espacios para sostener la propia formación del capital. En su fase neoliberal, el escenario idóneo para hacerlo es la región metropolitana, pues en ella se puede organizar y articular mejor la producción y los mercados internacionalizados (Soja, 2000). Dada la desintegración vertical de los procesos productivos, la región es la configuración espacial acorde con las nuevas necesidades de colaboración, creación de confianza y subcontratación que se establece entre los corporativos y todo el elenco de firmas que se constituyen en sus colaboradoras (Bridge \& Watson, 2003).

Ahora bien, las regiones metropolitanas, al presente, pierden la autonomía que otrora tuvieran al depender de sus conexiones con los procesos de la producción y de la especulación globales (Amin \& Malmberg, 1994). Como Massey ha llegado a sostener, para determinadas ciudades globales como Londres son más importantes los vínculos que consigue mantener con el resto de escenarios productivos y especulativos internacionales que los que sostenga con el resto de territorios de sus estados nación (Massey, 2008). De esta forma, el crecimiento o desarrollo del capital en determinadas regiones metropolitanas depende más de su éxito en cómo se vinculan a los demás espacios que conforman el capitalismo neoliberal de orden global (Vázquez, 2004). Desde esta vinculación a las inversiones internacionales, no sorprende observar acervas competencias entre regiones y ciudades para ofrecer las mejores condiciones que garanticen una más ampliada acumulación del capital (Sassen, 2000).

El entorno urbano se convierte entonces en un escenario ideal para observar aquellos procesos de neoliberalización que enfatizan Peck, Brenner y Theodore. Así, más allá de asentar la importancia del territorio para la formación del capital, se presentan continuación algunas formas en las que tiene lugar dicho asentamiento para evaluar las consecuencias que acarrea para la movilidad urbana.

\section{Manifestaciones de la neoliberalización del territorio y su incidencia en la movilidad urbana}

¿De qué formas concretas tiene lugar la neoliberalización del territorio y cómo inciden en el fenómeno de la movilidad urbana? En este apartado se intentarán establecer algunas de las características que adquiere el medio construido bajo el período neoliberal y los retos que le plantean a la gestión de la movilidad urbana. territarias 32

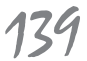




\subsection{Las deslocalizaciones y desinversiones en el territorio}

El neoliberalismo supuso una aguda reestructuración de las actividades económicas a escala planetaria. La supeditación de la economía a sus dimensiones financieras implicó que muchas de las ciudades del occidente desarrollado cambiaran radicalmente su especialización productiva, dejando de ser puntales mundiales de la industrialización para concentrarse en el sector de los servicios a la producción, la contabilidad y las finanzas. En ciudades como Londres (Massey, 2008) o Los Ángeles (Soja, 2000), los antiguos cinturones industriales se desmoronaron rápidamente ante la caída de beneficios en el sector y la deslocalización de estas actividades a países en donde los costos productivos eran más bajos. El territorio de estas ciudades quedó tensionado por un panorama de fábricas cerradas, fugas de capitales e instalaciones abandonadas (Harvey, 1994). A su vez, las poblaciones trabajadoras se encontraron repentinamente desempleadas y viviendo un proceso de descualificación al no encontrar trabajos acordes a sus capacidades. Buena parte de estas industrias, en un contexto internacional que suprimía las restricciones para el movimiento de los capitales y las inversiones (Harvey, 2005), fueron reinstaladas en los nuevos países industrializados (Soja, 2003), en enclaves no necesariamente integrados territorialmente con los entornos urbanos tradicionales, sino ubicados en nodos de comunicaciones y transportes estratégicos para la movilización de mercancías.

En ambos tipos de países, los desarrollados y aquellos que se encuentran en vías de desarrollo, el neoliberalismo modificó el guión que habrían de seguir las instituciones públicas hacia una mayor restricción presupuestaria y una reducción de las políticas redistributivas. El que la competencia fuera la nueva forma de relación entre las regiones del mundo hizo que las municipalidades vieran sus propios territorios no como espacios para planificar en busca de un desarrollo homogéneo y equilibrado, sino como la oportunidad de generar nuevos recursos y establecer combinaciones más eficientes de los distintos factores productivos para la llegada de las inversiones exteriores (Baeten 2012; Brenner, 2003; Zetter, 2004). El objetivo de los gobiernos dejó de centrarse en las poblaciones marginadas y en riesgo de exclusión, frente a la necesidad de atraer nuevos inversores. Estas reestructuraciones han motivado a que las dinámicas internas de las ciudades en desarrollo y de las desarrolladas cambien sustancialmente, lo que impone nuevos retos para la gestión de la movilidad que habría que estudiar pormenorizadamente.

En particular, en las ciudades desarrolladas se está por ver de qué forma los tradicionales sistemas de transporte se han ajustado o incluso han contribuido a estos fenómenos de desinversión industrial, lo que deja cada vez más confinados los antiguos barrios en los cinturones industriales y obreros. Así, en el contexto de las ciudades territarios 32 140 
británicas y norteamericanas, se ha estudiado la forma en la que las desinversiones en la red de autobuses que anteriormente servía a estas áreas ha contribuido a acelerar su decadencia (Lucas, 2004). Sin embargo, se necesita un programa más detallado de investigación, sensible a los distintos contextos nacionales y regionales, que siga la pista de cómo se han entrelazado las desinversiones productivas con las (des) inversiones en los sistemas de transporte en estas regiones. En esa dirección, se debe continuar en la línea de investigar cómo la privatización de los servicios de transporte público abona a la desconexión territorial de aquellos sectores más dispersos, con peor acceso o de menores recursos. ${ }^{3}$

En el mismo contexto desarrollado, este programa debe enriquecerse además de estudios que indaguen cómo las presiones neoliberales por el control de los déficits públicos han comportado una reconsideración de las políticas de movilidad urbana y han incidido en la ya mencionada desinversión y decadencia de los espacios urbanos. Las investigaciones sobre las intervenciones públicas concretas en los sistemas de movilidad deben ser capaces de leer la existencia o no de ese cambio de paradigma, de forma que se pueda encontrar evidencia, por ejemplo, del papel que están cumpliendo las más recientes inversiones en infraestructura para la movilidad en la rehabilitación y los procesos de mercantilización y gentrificación urbanos, o en la puesta en valor de nuevos movimientos especulativos en los suburbios. ${ }^{4}$
Por lo que hace a los países en desarrollo, se abre una agenda de investigación muy fecunda con el estudio de cómo se reorganizan los sistemas de transporte para cubrir las necesidades de los nuevos emplazamientos de la industrialización internacionalizada, como la apertura de múltiples zonas francas. ${ }^{5}$ A este respecto, resultará muy revelador el poder ubicar la existencia de varios subsistemas, uno focalizado a la más fácil y eficiente movilización de recursos productivos para los referidos emplazamientos y otro tradicional, encargado de servir las necesidades de movilidad de las poblaciones locales. En estos contextos, es muy importante atender a las particulares combinaciones entre esfuerzos públicos, privados formales y privados informales en la provisión de servicios de movilidad. Dichas investigaciones servirán para comprender mejor las dinámicas neoliberales, en especial cómo la gestión pública del transporte se realiza en pos de reforzar los procesos de acumulación de los capitales internacionales en los nuevos emplazamientos productivos, o se recupera una visión integradora sobre el territorio que intenta favorecer la inclusión de los diferentes espacios de las ciudades.

Al mismo tiempo, se suscita la hipótesis sobre el sentido puntual y no sistémico de las inversiones en movilidad. En la lógica neoliberal, y siguiendo cálculos sobre rendimientos, niveles de ocupación y aforos, se corre el riesgo de que se produzcan intervenciones dispersas y es posible que hasta inconexas, dejando a sectores importantes
${ }^{3}$ Como han hecho trabajos como los de Gwilliam (2013) o Lukas (2004).

${ }^{4}$ Como confirman trabajos como los de Zhang y Wang (2013) o Bocarejo, Portilla y Pérez (2013).

${ }^{5}$ Así Sánchez Puín (2011), para el caso de Bogotá, se ha encargado de poner de manifiesto la relación entre las distintas reformas del aeropuerto El Dorado con el desarrollo de las actividades industriales de la Zona Franca Fontibón.

territarios 32 141 
- Tal es el desarraigo de buena parte de la planta productiva mundial, que algunos autores hablan, más que de un mundo de lugares de factorias, de un mundo de flujos manufactureros (Audirac, 2003).

${ }^{7}$ Gifford (2003) ofrece una revisión de varios casos de vias dentro del sistema de autopistas interestatales estadounidense.

\section{territarias 32} 142 de la población con escaso servicio y espacios de la ciudad desarticulados e inmovilizados. Esta posibilidad ha hecho que se hable del sentido urbano, pero no metropolitano y global (Figueroa, 2005), de estas intervenciones que contribuirían al proceso de aislamiento y desconexión del territorio.

En el contexto global, más allá de estas reestructuraciones territoriales de la producción y de sus consecuencias para los tradicionales sistemas de transporte, el nuevo periodo histórico comporta una más profunda y radical inestabilidad territorial. La inestabilidad de los mercados de capitales y la inducción al cambio de gustos y preferencias en los mercados de bienes y servicios inaugura una nueva etapa en la que la planificación de la producción se hace muy incierta y riesgosa (Swingedouw, 2004). De este modo, las corporaciones siguen una estrategia consistente en establecer vínculos cada vez más laxos y tenues con los diversos territorios, calculando que los costos sean lo más bajos posibles en el momento en que tengan que desinstalar sus enclaves productivos en busca de nuevos emplazamientos. ${ }^{6}$ En un mundo que alienta la movilidad de capitales, cuando los inversores encuentran resistencias o rigideces bajo la forma de regulaciones laborales, administrativas o ambientales, su respuesta inmediata es la desinversión y la búsqueda de nuevos entornos con climas más amigables para sus negocios (Bauman, 2010). La revolución tecnológica en las comunicaciones y procesamiento de la información que sirve de soporte al neoliberalismo facilita las actividades de coordinación y gestión de esta serie de emplazamientos productivos, territorialmente cada vez más inestables (Boltanski \& Chiapello, 2005).

Si las ciudades han sufrido un proceso de transformación económica y profunda y si están llamadas a sufrir cada vez más acelerados procesos de inversiones y desinversiones, los sistemas de transporte están llamados a experimentar asimismo importantes cambios. Los elevados costes de inversión en infraestructuras en autopistas o corredores de transporte masivo corren el riesgo de ser irrecuperables, al producirse cambios en la demanda que los condenen a la subocupación, apenas construidas dichas infraestructuras. ${ }^{7}$ A tal efecto, en el último decenio se han puesto en marcha medidas de provisión más flexibles, de los servicios de transporte, bajo el esquema Demand-ResponsiveTransport (Mulley \& Nelson, 2009). De igual modo, se necesitan estudios de caso más precisos que analicen cómo está respondiendo la movilidad urbana a estas situaciones de mayor incertidumbre territorial, forzadas por los procesos flexibles de inversión propios del neoliberalismo.

\subsection{El territorio como espacio para la especulación}

Ya se comentó acerca del papel que ocupaba el sector inmobiliario, a juicio de Harvey (1994), dentro del desarrollo de la acumulación capitalista bajo el neoliberalismo. Frente al riesgo de que una sobreinversión en el circuito primario de la producción 
acarreara las conocidas crisis de superproducción y de precipitación de los rendimientos, aparecía un segundo circuito para las inversiones, el del sector inmobiliario, que cobraba un papel estabilizador y aumentaba las opciones para la obtención de beneficios. ${ }^{8}$ Dentro del neoliberalismo, el inmobiliario se convierte así en un nuevo espacio de especulación que somete al territorio y a la gestión de la movilidad a nuevos retos y tensiones.

Este hecho se ubica en el contexto, además, de la internacionalización de los mercados de capitales, de manera que el destino del territorio de las ciudades se juega cada vez más en ámbitos globales. En los países desarrollados, cada vez es más notoria la presencia de capital internacional detrás de los principales proyectos de rehabilitación y desarrollo urbano (Soja, 2000). $\mathrm{Al}$ mismo tiempo, los países en desarrollo se afanan por generar las mejores condiciones posibles para la llegada de dicho capital (Zetter, 2004). En ambos contextos, la fórmula que han seguido los gobiernos municipales para convertir el espacio urbano en un instrumento para la especulación capitalista internacional es bien conocida y replicada: desregulaciones, privatizaciones, austeridad fiscal, liberalizaciones, eliminación de impuestos a intermediaciones, impulso a la mercadotecnia de los espacios, control y vigilancia de poblaciones pobres, etc. (Peck, Theodore \& Brenner, 2009).

Sin lugar a dudas, en este proceso de valorización mercantil del territorio la movilidad urbana y los sistemas de transporte han representado un papel muy importan- te, aunque insuficientemente reconocido. En este tenor, deben reforzarse las investigaciones que estudien cómo ciertas mejoras en los sistemas de transporte, como la extensión y apertura de nuevas autopistas, o la inauguración de líneas de trenes o suburbanos dirigidos a las clases medias y altas, han impulsado la especulación sobre los territorios adyacentes y el incremento de los rendimientos inmobiliarios. ${ }^{9}$ Estas investigaciones deben ser extremadamente sensibles a los contextos nacionales y urbanos propios. Se requiere de mucha investigación empírica que pueda poner de relevancia la aparición de concretos modelos de acumulación y que reparen en elementos como los siguientes: (a) los tipos y características del sector urbano rehabilitado, (b) las soluciones de movilidad que impulsan o sostienen esos procesos de rehabilitación (c) la composición del capital involucrado entre capitales públicos, privados locales o privados internacionales, (d) los procesos intervención o cooptación de las administraciones públicas que pongan en evidencia su papel dentro de la promoción empresarial de los proyectos urbanos, (e) los niveles de obsolescencia previstos de las instalaciones y la infraestructura y el seguimiento al inverso proceso de desinversiones, etc. El atender a estos elementos ayudará a concretar qué particular régimen neoliberal está en juego, y qué vías concretas de producción del espacio y de la movilidad está tomando.

Ahora bien, no solo debemos estar atentos a la dimensión local de las inversiones urbanas y en movilidad. Bajo el neoli-
${ }^{8}$ En Estados Unidos se ha documentado que el $1 \%$ de la población más rica obtiene el $45 \%$ de susbeneficios de las inversiones inmobiliarias (Marcuse, 273).

9 Así, por ejemplo, lo reconoce Wolfe (2010) para el caso de Brasil, o Zhang y Whang (2013), para el caso de China. tersitarios 32

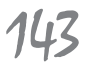


beralismo, el territorio es producido para su exhibición y promoción en una escala igualmente internacional. Es muy abundante la literatura que advierte de la gestión planificada de los procesos de decadencia y de rehabilitación en el seno de las principales urbes (Esser \& Hirsch, 1994). Buena parte de ellos tienen lugar en espacios céntricos empobrecidos (Hackworth, 2007) y su finalidad es remodelarlos y reconvertirlos profundamente, gentrificarlos de forma que se pongan a disposición de las clases medias y altas (Mitchell \& Beckett, 2008) y se refuercen los mecanismos de la valorización del espacio. La novedad del periodo neoliberal es que se abre y se profundiza un nicho de mercado, cada vez más internacional, de consumidores de este tipo de espacios. Las élites de negocios y financieras, los sectores de profesionales cualificados $y$ de ejecutivos comienzan a organizarse internacionalmente, de manera que las ciudades deben producir su territorio con vistas, cada vez más, en sus gustos y preferencias de hábitat. Y al igual que se internacionalizan los residentes más acaudalados de las principales ciudades globales, otro tanto ocurre con sus visitantes y turistas. Esta internacionalización de los hábitats y los gustos urbanos ostentosos genera una especie de intercambiabilidad de cualquier espacio urbano mundial (Blomley, 2004), que acarrea, eso sí, la exclusión de poblaciones locales pobres que pudieran romper con ese estilo de altos consumos.

No cabe duda de que en esta estrategia territarias 32 144 de especulación y mercantilización internacional de la ciudad, la gestión de los accesos y la movilidad a estos espacios producidos es de gran importancia. Para movilizar internacionalmente los gustos de esta clase dirigente mundial es fundamental que las ciudades extiendan las redes y los transportes necesarios (Peck, Theodore, Brenner, 2009), dentro de ese mismo estilo del alto consumo. En este terreno, se precisan de otros tantos estudios de orden espacial y cualitativo que se centre en, al menos, tres áreas de investigación. Dada la naturaleza global de las dinámicas señaladas, estos estudios son pertinentes en espacios desarrollados y en vías de desarrollo. Así, en primer lugar, debe analizarse cómo las recientes intervenciones en la movilidad urbana, favoreciendo tanto el transporte privado como el masivo de altos estándares, puedan dirigirse a estos sectores de la élite internacional. En segundo lugar, debe analizarse cómo se pueden usar estas intervenciones en la movilidad urbana para segregar y para invisibilizar a todas aquellas otras poblaciones locales que rompen con el aire de estilo de los altos consumos. Finalmente, ha de estudiarse también de qué forma la imposición de ciertos estándares internacionales en la provisión de los servicios de movilidad (BRTs, metros, aeropuertos, trenes) puede vincularse no tanto con la solución de problemas concretos y locales, sino con la imposición de unos patrones de uso del transporte, acordes con las competencias y habilidades de la referida élite extraterritorial.

Ahora bien, la ampliada especulación sobre el territorio urbano presente bajo el neoliberalismo no se circunscribe a la pro- 
ducción de los espacios de las clases altas. En el ámbito latinoamericano, como señala Luis Felipe Cabrales (2010), muchas de las soluciones habitacionales de las clases populares que antaño se basaban fundamentalmente en la auto-construcción han sido recientemente mercantilizadas y ubicadas al interior de los circuitos de especulación urbana. De este modo, crece el espacio y las oportunidades para ampliar la acumulación capitalista en la ciudad. Dichas inversiones se ubican en este contexto de desregularización neoliberal del espacio urbano, lo que suele motivar que en muchas ocasiones, y en los países en desarrollo, se materialicen en espacios en los que la reconversión de suelo agrario a suelo urbano multiplica la previsión de los beneficios. La resultante son conjuntos habitacionales de cientos, miles y en ocasiones decenas de miles de viviendas de muy escasa calidad y ubicados en la periferia de la ciudad (Jacquin, 2012), en ocasiones incluso fuera de la trama urbana. Así, una actividad que en las ciudades en desarrollo, de las décadas del cincuenta al ochenta, se dejaba a la suerte y se ponía en las manos de las propias poblaciones pobres, ahora se mercantiliza y ofrece una nueva oportunidad de ampliar la especulación en la ciudad. Además, el que un motivo para su construcción sea la disponibilidad de suelo rural barato y fácilmente recalificable hace que el crecimiento urbano en estos últimos decenios haya tenido una naturaleza tremendamente dispersa y fragmentada (Eibenschutz \& Carrillo, 2011). En ocasiones, y ante la referida desregulación, no hay más lógica para la planeación de estos conjuntos habitacionales que el cálculo de las oportunidades de beneficio. El lugar donde se hallen, y su conexión con la red de servicios urbanos, no son aspectos que se tomen en consideración. ${ }^{10}$

Esta total desregulación y apertura a la especulación sobre el territorio de las ciudades de los países en desarrollo genera problemas de movilidad muy notorios y evidentes. Se necesita una amplia base de estudios que se enfoquen, fundamentalmente, en abordar estas tensiones que ocasiona la neoliberalización del territorio sobre las formas de la movilidad urbana de las poblaciones pobres. Por un lado, deben estudiarse las estrategias de movilidad que siguen los habitantes de estas poblaciones periféricas para llegar a sus centros de trabajo y a los servicios y para organizar la producción y reproducción de sus hogares. ${ }^{11}$ Por otro lado, y con una importancia crucial para la gestión futura de la movilidad de estas ciudades, deben investigarse qué soluciones se están proponiendo y cómo se están reacomodando los sistemas de transportes para satisfacer las necesidades de movilidad de estas poblaciones tan periféricas. Finalmente, y en un orden fundamentalmente cualitativo, deben investigarse las formas en las que las clases populares así desplazadas se apropian de estos territorios periféricos y discontinuos, y qué sentido dan a una cotidianidad instalada en el confinamiento y la desconexión respecto de lo urbano. ${ }^{12}$
${ }^{10}$ Esos problemas de conectividad solo aparecen en el escenario de la toma de decisiones de las inmobiliariascuando son tan notorios que los nuevos inquilinos comienzan a abandonar sus nuevas viviendas por las dificultades para llegar a los servicios y sus trabajos. Según diversas entrevistas realizadas en el sector, en el Área Metropolitana de Guadalajara, en estos casos la existencia de viviendas deshabitadas se convierte en una fuente de intranquilidad e inseguridad que obstaculiza la llegada de nuevos vecinos y las propias ventas.

${ }^{11}$ En esta línea apunta el propio estudio de Jacquin (2012).

${ }^{12}$ En este sentido apuntan los brillantes trabajos de Lindón $(1997,2006)$.

territarios 32

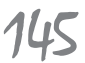




\subsection{Neoliberalismo, polaridades territoriales y movilidad urbana}

Alguna de las características que se han presentado antes sobre los procesos de neoliberalización del territorio bastaría para que se dedicara toda la atención a observar cómo afectan a la movilidad urbana y a los sistemas de transporte. Resumiéndolas en un concepto integrador, acuñado para el ámbito desarrollado estadounidense, se podría recurrir a aquel término que propone Soja (2000) para comprender la ciudad neoliberal: exopolis. Es decir, un espacio turnedinside-out, por la fuerte urbanización de los suburbios y la consolidación de centros de trabajo, oficinas y centros comerciales y de ocio periurbanos, pero, al mismo tiempo, un espacio turnedoutsidein en el que la periferia y la marginalidad, se instalan como determinantes del centro de la ciudad contemporánea. Así, un primer y más general programa de investigación consistiría en examinar la forma en la que los medios de comunicación y transporte contemporáneos han posibilitado esta reestructuración de la ciudad, es decir, conocer la base tecnológica e infraestructural para la aparición de la nueva geomorfología de enclaves corporativos dispersos, parques de negocios y sedes administrativas en las periferias, o centros comerciales y resorts de lujo ubicados igualmente en los confines de la ciudad (Cervero, 2004).

Ahora bien, lo realmente destacado de esta descripción es su capacidad para expresar, al lado de la ruptura de la ciudad, la aparición de intensas desigualdades sociales y espaciales dispuestas en régimen de estricta coespacialidad. No en vano, esa manera en la que la periferia se adentra en el seno de la ciudad tradicional resume la forma en la que lo que tradicionalmente se llamaba el Tercer Mundo penetra hasta el corazón de las urbes que con anterioridad eran consideradas puntales del Primer Mundo. El fenómeno no es gratuito, sino que responde justamente a esa tendencia hacia la desregulación propia del neoliberalismo, que favoreció la caída generalizada de los costos productivos y que hizo que se instalaran condiciones laborales y de reproducción de la clase obrera propias de los países pobres en el seno de las ciudades prósperas (Gough \& Eisenschitz, 2006). Como consecuencia, diversos autores (Audirac, 2003; Sassen, 2000) sostienen que la referida distinción entre el Primer y el Tercer Mundo es insostenible en la actualidad, dada la gran heterogeneidad y desigualdad de las geografías productivas presentes en la gran mayoría de las ciudades del mundo. Por ello, habrá que estudiar con detenimiento cómo se expresa esta polarización en las ciudades neoliberales y de qué manera se relaciona con los fenómenos de movilidad urbana y con los sistemas de transporte.

Merece la pena ubicar la discusión en el contexto ligeramente más amplio en que la ubica Bauman (2003), al describir una nueva geografía del poder en una escala planetaria. Dentro de los procesos de desregularización a los movimientos del capital, bajo su juicio, el recurso que en la actualidad construye la posición de la clase alta es su nueva capacidad para desterritorializarse, 
evadirse sin responsabilidad alguna de los emplazamientos ocupados tan pronto como estos dejan de ser productivos o dejan de rendir beneficios (Bauman, 2003). Las antiguas rigideces, propias del estado fordista keynesiano, que tomaban la forma de los seguros por desempleo, las regulaciones sobre los despidos o las negociaciones colectivas, fueron progresivamente eliminadas en la primera fase neoliberal del repliegue, lo que permitió al capital y a los capitalistas moverse más libremente a lo ancho del planeta. Así, en la actualidad, la clase alta ha perdido las razones que la vinculan moralmente a la ciudad (Bauman, 2003), más allá de la corta duración de los cada vez más vertiginosos ciclos de las inversiones y las desinversiones.

El escaso compromiso con el lugar que desarrollan las élites internacionales tiene la contrapartida de la multitud, vinculaciones y relaciones transterritoriales extendidas en toda la red de ciudades globales, hasta el punto en que se establece una especie de estandarización internacional de los espacios para las élites a lo ancho de todo el mundo. Como han notado Bridge y Watson (2003), en la ciudad neoliberal estos espacios del poder y sus clases sociales suelen estar más estrechamente vinculadas con otros espacios similares en ciudades a lo largo de todo el globo que con los espacios inmediatamente vecinos de los centros empobrecidos o los antiguos cinturones industriales decadentes.

Esta movilidad translocal no es gratuita. Es necesario reiterar que proviene de la desregulación neoliberal sobre las tran- sacciones económicas y financieras a escala planetaria y que ha inaugurado un nuevo periodo de acumulación. Así, los grandes epicentros del poder monetario y financiero en el contexto desarrollado, como Nueva York, Londres o Tokio, para poder vincularse exitosamente en empresas de inversión y desinversión en todo el planeta (Brenner, 2003), tienen al mismo tiempo que independizarse de su tradicional sujeción al estado nacional fordista keynesiano, que se expresaba en toda una serie de trabas como los planes de desarrollo, los sistemas impositivos y la política redistributiva, o la existencia de aranceles a la exportación o importación de capitales.

No puede dejar de señalarse que esta nueva geografía del poder, que subraya las nuevas potencialidades de la movilidad, pero que pudiera parecer demasiado abstracta, debe materializarse en investigaciones más concretas y que descubran las bases infraestructurales que permiten y alientan esta extremada movilidad de las clases altas presentes. En particular, y según la inspiración de parte del trabajo de Hiernaux y Lindón (2004), debería examinarse el tipo de desplazamientos de la nueva burguesía neoliberal, sus modalidades o los espacios trasnacionales que configuran su especial geografía de la movilidad. Una buena cantidad de estudios empíricos han de dar forma a lo que en principio no son más que las lógicas coincidentes del neoliberalismo con la expresión de las movilidades concretas en las ciudades actuales.

En particular, las investigaciones deben centrarse en la forma en la que los sistemas territarias 32

147 
${ }^{13}$ Las presiones de estasélites a los gobiernos para que, por medio de los ejes carreteros, les ayuden a construir sus espacios privatizados, han sido también destacadas por Audirac (2003) para el caso de Guadalajara.

${ }^{14}$ Así lo alcanza a precisar Baeten (2012).

\section{territarias 32} 148 de transporte existentes permiten las movilidades urbanas segregacionistas de las clases triunfadoras del capitalismo neoliberal. En la escala urbana de los países en desarrollo, abundan los análisis (Cabrales, 2002; Calonge, 2013) sobre cómo estas clases se aíslan y confinan en los crecientes espacios de la riqueza de los fraccionamientos cerrados o gated communities. Asimismo, hay señalamientos sobre cómo se construyen sus complejos de ocio y de trabajo, bajo la premisa de la separación del más general ambiente urbano por medio de accesos limitados a los peatones o espacios integrados por corredores y vestíbulos semiprivatizados (Christopherson, 1994; Marcuse, 2003). Se necesita, sin embargo, incidir en la forma en la que estas clases sociales construyen sus movilidades para obtener la referida independencia respecto del espacio urbano y forzar su segregación en los puntuales y diseminados espacios para la élite.

En esta dirección, y en este mismo entorno en desarrollo, existen estudios dispersos que examinan el sentido segregacionista de algunas intervenciones en los sistemas de transporte en las ciudades contemporáneas. Así, se ha mostrado (Sohn, 2011) cómo, con intervención pública, se han construido infraestructuras de transporte que ayudan a que las élites consigan conectarse por medio de sus dispersas ciudades privatizadas en la Ciudad de México. En un nivel de mayor complejidad, Rodgers, Beall y Kanbur (2011) hablan para el caso de Managua no tanto de comunidades cerradas sino de redes fortificadas para referirse a la extensión de una red privada de vialidades y autopistas de alta capacidad que ayudan a las élites de la ciudad a desplazarse por sus espacios independizados del poder. ${ }^{13} \mathrm{En}$ cualquier caso, la mirada sobre la red de transportes ha de cualificarse para evidenciar no solo las formas de conexión, sino también las de desconexión que impiden el acceso del resto de las poblaciones a los medios de transporte y a los espacios para las élites neoliberales. ${ }^{14}$ Por tanto, existe un enjundioso trabajo por delante para acumular más evidencia y comenzar a precisar posibles tipologías y fórmulas contextualmente específicas de cómo las clases altas construyen sus movilidades segregadas $\mathrm{CO}^{-}$ mo sostén a sus esfuerzos por mantener su poder extraterritorial. Este esfuerzo habrá de ser contextualizado en la particularidad económica e institucional de cada contexto de investigación, con la intención de realizar estudios comparativos.

Este tipo de investigaciones, sin embargo, no puede ignorar la situación en que quedan el resto de clases sociales en un contexto de gran polarización. En un orden mundial, si lo que permitía a las élites sostener su poder eran sus ampliadas posibilidades de movilidad, lo que explique la supeditación de las clases bajas va a ser su confinamiento e inmovilidad sobre unos territorios específicos amenazados por la desinversión y la decadencia (Bauman, 2003). Este confinamiento está compuesto por una serie de fenómenos entrelazados de los que es difícil escapar. En primer lugar, una buena parte de los espacios habitados por las clases populares se encuentran desconectados respecto de las redes de acumu- 
lación y poder que constituyen los puntales de la economía internacionalizada (Sassen, 2000). En segundo lugar, y de una forma creciente, dichos espacios están también desvinculados de su propia realidad urbana, de las otras ciudades habitadas por las élites extraterritoriales (Rodgers, Beal \& Kanbur, 2011). Finalmente, ante estas clases no solo se extienden barreras y desconexiones físicas, sino también políticas y morales. En su mayor parte, como ciudadanos de segunda clase, les son negados sus derechos a la movilidad en el momento en que, al calificárseles como emigrantes, refugiados o indocumentados, se les impone toda la impenetrabilidad de la frontera (Sibley, 1995).

En todo el orbe, las poblaciones pobres, compuestas por migrantes, por antiguos obreros afectados por los cierres de fábricas, por una generación enfrentada a la flexibilización y precarización de la mano de obra, pero también por contingentes instalados en la informalidad, se sitúan así en los centros abandonados de las ciudades, en sus cinturones industriales en decadencia, o en los nuevos espacios que la especulación inmobiliaria les ha deparado como conjuntos habitacionales en la periferia. En ocasiones, la simple fragmentación urbana es la que produce su confinamiento. En otras más hirientes, se superpone un control explícito de las fuerzas de seguridad que evita que aparezcan por los espacios mercantilizados para las élites internacionales (Davis, 1990).

Pero comoquiera que se produzca esta reclusión de los sectores populares de las ciudades mundiales, el papel que desem- peñan los sistemas de transporte puede ser fundamental para explicar su particular (in) movilidad urbana. Así, al mismo tiempo que habían de investigarse las formas en las que dichos sistemas garantizaban la huida de las clases altas hacia las redes globales de la acumulación, el estudio inverso tiene que seguirse respecto a las clases bajas. En particular, se debe poner especial atención no solo en las dificultades de acceso de estas clases a los sistemas de transporte, sino en las deficiencias y en los obstáculos que presentan en términos de cercanía, costo, conveniencia o flexibilidad. Como se ha mostrado, para el caso de Copenhague en el contexto desarrollado (Baeten, 2012), es posible que buena parte de su confinamiento sea reforzado por unos sistemas de transporte que solo les permiten la movilidad hacia las oportunidades educativas y laborales que comportan bajos salarios, inestabilidad laboral y precarización de las condiciones de vida.

Esta línea de investigaciones debe prestar una especial atención a la articulación de la red de transporte público. Dado que la mayor parte de inversiones en infraestructura de transporte consiste en infraestructura vial para el automóvil privado, se debe estudiar qué opciones de transporte público restan para todas aquellas clases que no pueden permitirse adquirirlo. En estas circunstancias, no hay que olvidar el rápido deterioro a que se enfrenta esta provisión pública de los servicios de transporte en pleno contexto neoliberal. Como bien ha señalado Figueroa (2005), para el caso de los países en desarrollo. territarias 32 149 
[...] el transporte urbano se ve confrontado con varias situaciones críticas: por un lado, debe asimilar e integrar los cambios que se definen para las políticas públicas del transporte público, especialmente las que ponen el acento en la liberalización; por otro lado, debe enfrentar una aguda competencia que se origina en un mayor uso del automóvil privado y de formas artesanales de transporte público; y por último, debe habituarse a nuevas estructuras de viajes y a la cobertura de áreas urbanas más extensas, pero con menor densidad de demanda (42).

Miradas en su conjunto, las polaridades urbanas de la ciudad neoliberal deben enseñar a contemplar las heterogeneidades, multiplicidades y jerarquizaciones sociales inscritas en el mismo espacio. De esta forma, las profundas desigualdades retratadas serían el reflejo de una construcción material de la diferencia espacial y de la red de movilidades que rompe con la homogenidad espacial y social a la que aspiraba el anterior estado fordista y keynesiano (Manderscheid, 2009). En otras palabras, ya no se estaría ante el espacio homogéneo y continuo de una ciudad(anía) integradora, sino ante un espacio en sí mismo estriado, diferencial, que escinde muy diversas condiciones sociales y posibilidades de integración. Los sistemas de transporte, en sí mismos heterogéneos y múltiples, están contribuyendo a generar esta polarización social y espacial. Como la propia Manderscheid (2009) afirma: “debido a que las personas, el capital y las cosas no se mueven por las mismas rutas y con ritmos similares es que aparecen diferentes espacialidades que se presentan incluso menos congruentes con los pasados territorios sociales".

El reto entonces es emprender investigaciones particulares que muestren cómo la heterogeneidad propia al sistema de transporte genera ese espacio estriado, las polidaridades territoriales y todas las formas de desigualdad social que albergan las ciudades contemporáneas. Así, cabría observar cómo la inversión en vialidades puede favorecer el propio aislamiento $\mathrm{y}$ la huida del espacio cercano de las élites trasnacionales (Redshaw, 2008), cómo se produce también una gentrificación de una parte del transporte público que comunica rápidamente, pero a precios prohibitivos, las ciudades privatizadas (Mason, 2007) o cómo las redes de autobuses, que sufren los efectos de las desinversiones públicas y del deterioro, confinan inadvertidamente a las clases bajas en sus espacios desconectados y decadentes (Cresswell, 2006).

\section{Conclusiones}

Corresponde ahora sintetizar brevemente algunas de las advertencias sobre cómo tendría que configurarse una agenda de investigación que estudiara las relaciones entre la neoliberalización del territorio, la manifestación de las movilidades urbanas y la gestión de los sistemas de transporte.

En primer lugar, están todas las preguntas relacionadas con la forma en la que la neoliberalización del territorio ha tensionado los sistemas de transporte existentes. En este tenor se deben intentar seguir los 
cambios que el sistema neoliberal ha forzado en el entorno urbano. Fundamentalmente, deben resaltarse los movimientos de reestructuración del territorio, derivados de los movimientos de deslocalización y relocalización industrial, de la aparición de nuevos centros de trabajo en posiciones excéntricas o de la instalación de centros de servicios y de ocio, igualmente en zonas periféricas de la ciudad. Este registro de cómo las actividades productivas del neoliberalismo vienen a romper y a fragmentar la ciudad será de especial interés para comprender la reestructuración y relocalización de la demanda para los antiguos sistemas de transporte. Aquí habrá que estudiar, según ámbitos desarrollados o en desarrollo, cómo estos sistemas han ido adecuándose a los cambios del espacio urbano consignados y qué estrategias han seguido las distintas capas de la población para desplazarse a su interior.

En los países en desarrollo, es fundamental, además, advertir cómo se ha producido la instalación de emplazamientos productivos internacionalizados como las zonas francas. Un campo de estudio especialmente fecundo es observar la posible aparición de distintos sistemas de transporte superpuestos, uno privilegiado para la movilización de mano de obra y para la movilización de insumos y de mercancías, y otro subdesarrollado que sirva a las necesidades cotidianas de las poblaciones vinculadas a estos emplazamientos productivos. De igual forma, y siguiendo con las poblaciones populares, es de máxima importancia estudiar la posible dispersión de sus hábitats periféricos, las dificultades que han tenido los sistemas de transporte para movilizarlos exitosamente y las estrategias, competencias e identidades de estas poblaciones alojadas en estos contextos desconectados y periurbanos.

Ahora bien, los sistemas de transporte no solo han aparecido como estructuras pasivas que se ajustan y responden a los cambios que se producen en el territorio neoliberalizado. También son ellos mismos agentes de transformación y de cambio del espacio urbano, y protagonistas importantes en el proceso de neoliberalización del territorio. En este orden de ideas, el recuento ofrecido sugiere la necesidad de investigar cómo los propios sistemas de transporte han alimentado los procesos de desinversión y de reinversión territorial. En particular, una línea de investigación se centra en comprobar cómo la falta de recursos para el transporte público y la decadencia de su infraestructura y vehículos acarrea un agravamiento de la desconexión y la decadencia de los territorios en declive. Otra consiste en observar las dinámicas inversas, es decir, cómo ciertas intervenciones en los sistemas de transporte, como la apertura de nuevas autopistas o líneas de ferrocarriles, metros o BRTs ayudan a poner en valor nuevos espacios, o sirven para potenciar las posibilidades de acumulación proyectadas para nuevos centros habitacionales, de trabajo o de ocio de las clases altas.

En esta medida, los sistemas de transporte pueden estarse constituyendo en nuevos vectores de polarización social y territorial, impulsando esta tendencia propia territarias 32

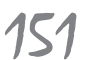


del neoliberalismo. Así, por el lado de las clases altas, una línea de investigación tiene que ser capaz de comprobar cómo los sistemas de transporte son proyectados para impulsar sus altas y amplias movilidades, tanto al interior de la región metropolitana como en su conexión con otras ciudades mundo. Pero, al mismo tiempo, debe observarse la forma en la que el diseño e instrumentación de los sistemas de transporte pueden estar sirviendo para la secesión de esta clase alta respecto de los tradicionales entornos urbanos, al generar efectos de barrera para el resto de poblaciones. Por el lado de las clases bajas, e íntimamente ligado con lo anterior, debe analizarse cómo los sistemas de transporte se transforman en factores de exclusión. En esta línea caben desde investigaciones de corte cartográfico, como otras de índole cualitativo que indaguen la formación de nuevas identidades del confinamiento y la subalternidad.

Pero la presentación ofrecida más arriba no solo ha puesto al descubierto las formas en las que los sistemas de transporte han sido modificados o han contribuido a modificar la neoliberalización del territorio. También se ha puesto de manifiesto momentos en que ellos mismos se neoliberalizaban. En este orden de asuntos, aparece todo un campo de investigación que examine cómo la gestión de la movilidad urbana pierde el enfoque global, integrador y sistémico que tenía bajo el período fordista keynesiano, para articularse por medio de intervenciones puntuales, ad hoc y fragmentadas. Se podría observar entonces cómo pasa de ser instrumento de coordinación e integración socio-espacial a convertirse en instrumento de promoción puntual de determinados proyectos de mercantilización del espacio urbano. A este respecto, habría de examinarse si no puede hablarse de un proceso de gentrificación de los propios sistemas de transporte. Aparte, también cabría investigar todo el rango de soluciones flexibles de los sistemas de transporte a una realidad territorial igualmente flexible. Se está hablando aquí de aproximaciones más circunstanciales y temporales a la solución de determinados problemas de movilidad como son los transportes sensibles a una demanda cada vez más difícilmente modelable. Por último, otro orden de fenómenos sobre la neoliberalización de los propios sistemas de transporte estaría relacionado con las formas en las que se generan nuevos patrones de movilidad internacionalizados y una serie de estandarizaciones de la infraestructura y operatividad a nivel internacional. Aquí cabrían aspectos desde la señalética, el diseño de conmutadores, vehículos e instalaciones, la gestión de los sistemas de información, o las formas de interconexión, entre unas modalidades y otras. Esta serie de estudios podrían estar inspirados por la hipótesis de si esas estandarizaciones tienen por objetivo solucionar problemas locales y concretos de movilidad urbana o estuvieran dirigidos más bien a ponerse al servicio de las expectativas, necesidades y competencias de movilidad de la élite global.

Se ubique donde se ubique la pregunta de investigación, el presente trabajo debe dejar clara una idea. Se debe pasar de hablar 
de un sistema de transporte que aspira a favorecer una accesibilidad universal y homogénea hacia los distintos espacios de la urbe, para comprenderlos en su dimensión plural, al promover la escisión de realidades sociales y espaciales diversas, heterogéneas y acusadamente desiguales. Si el territorio urbano bajo el neoliberalismo se encuentra profundamente fragmentado y estriado, una parte de los estudios sobre la movilidad urbana tienen que investigar la forma en la que los distintos subsistemas clasistas de transporte producen y participan esa fragmentación.

\section{Referencias}

Amin, A., \& Malmberg, A. (1994). Competing Structural and Institutional Influences on the Geography of Production in Europe. En A. Amin (Ed.), Postfordism (pp. 227-248). Oxford: Blackwell.

Audirac, I. (2003). Information-Age. Landscapes Outside the Developed World. Journal of the American Planning Association, 69(1), 16-32.

Baeten, G. (2012). Neoliberal Planning. Does it Really Exist? In G. Baeten \& T. Tasan-Kok (Eds.), Contradictions of Neoliberal Planning (pp. 205-213). London: Springer.

Bauman, Z. (2003). City of Fears, City of Hopes. London: Goldsmiths College.

Bauman, Z. (2010). La globalización. Consecuencias humanas. México: Fondo de Cultura Económica.
Blomley, N. (2004). Unsettling the City. Urban Land and the Politics of Property. London: Routledge.

Bocarejo, J. P., Portilla, I., \& Pérez, M. A. (2013). Impact of Transmilenio on density, land use and land value in Bogotá. Research in Transportation Economics, 40,78-86.

Boltanski, L., \& Chiapello, È. (2005). The New Spirit of Capitalism. London: Verso.

Brenner, N. (2003). La formación de la ciudad global y el re-escalamiento del espacio del Estado en la Europa occidental postfordista. EURE, Revista Latinoamericana de estudios urbanos regionales, 29(86), 5-35.

Brenner, N., \& Theodore, N. (2005). Neoliberalism and the Urban Condition. City, 9(1), 101-107.

Brenner, N., Peck, J., \& Theodore, N. (2010). After Neoliberalization? Globalizations, 7(3), 327-345.

Bridge, G., \& Watson, S. (2003). City economies. En G. Bridge \& S. Watson (Eds.), A Companion to the City (pp. 181-203). Oxford: Blackwell.

Cabrales, B. L. F. (2002). Latinoamérica. Paises abiertos, ciudades cerradas. Guadalajara: Universidad de Guadalajara.

Cabrales, B. L. F. (2010). El de atrás paga: el modelo metropolitano de Guadalajara. En O. Urquídez (Coord.), La reinvención de la metrópoli. Algunas propuestas (pp.75-96). Zapopan: El Colegio de Jalisco.

Calonge, R. F. (2013). Los sentidos de la ciudad. Sobre cómo mujeres y hombres territarias 32

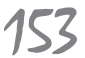




\section{territarias 32}

ordenan sus espacios vitales. Guadalajara: Universidad de Guadalajara.

Cervero, R. (2004). Job Isolation in the USA. Narrowing the Gap through Job Access and Reverse-Commute Programs. En K. Lucas (Ed.), Running on Empty. Transport, Social Exclusion and Environmental Justice (pp. 181-196). Bristol: Policy Press.

Christopherson, S. (1994). The Fortress City. Privatized Spaces, Consumer Citizenship. En A. Amin (Ed.), Postfordism (pp. 409-427). Oxford: Blackwell.

Cresswell, T. (2006). On the Move. Mobility in the Modern Western World. New York: Routledge.

Davis, M. (1990). City of Quartz. Excavating the Future in Los Angeles. London: Verso.

Eibenschutz, R., \& Carrillo, L. O. (2011). The Growth of Cities in Mexico. En T. Kaminer, M. Robles Durán \& H. Sohn, K. Robles \& Sohn (Eds.), Urban Asymmetries. Studies and Projects on Neoliberal Urbanization (pp. 84-97). Rotterdam: 010 Publishers.

Elliot, A., \& Urry, J. (2010). Mobile lives. London: Routledge.

Esser, J., \& Hirsch, J. (1994). The Crisis of Fordism and the Dimensions of a PostFordist Regional and Urban Structure. In A. Amin (Ed.), Postfordism (pp. 7198). Oxford: Blackwell.

Figueroa, O. (2005). Transporte urbano y globalización. Políticas y efectos en América Latina. EURE, XXXI(94), 41-53.
French, S., Leyshon, A., \& Wainwright, T. (2011). Finanzializing space, spacing financialization. Progress in Human Geography, 35(4), 798-819.

Gifford, J. (2003). Flexible Urban Transportation. London: Elsevier.

Gough, J., \& Eisenschitz, A. (2006). Spaces of Social Exclusion. London: Routledge.

Gwilliam, K. (2013). Cities on the move. Ten years after. Research in Transportation Economics, 40, 3-18.

Hackworth, J. (2007). The Neoliberal City. Governance, Ideology and Development in American Urbanism. London: Cornel University Press.

Harvey, D. (1994). Flexible Accumulation through Urbanization. Reflections on Postmodernism in the American City. En A. Amin (Ed.), Postfordism (pp. 361386). Oxford: Blackwell.

Harvey, D. (2005). Spaces of neoliberalization: towards a theory of uneven geographical development. Stuttgart: Franz Steiner Verlag.

Harvey, D. (2006). Neo-liberalism as creative destruction. Geografiska Annaler. Series B, Human Geography, 82(2), 145-158.

Harvey, D. (2007). A Brief History of Neoliberalism. Oxford: Oxford University Press.

Harvey, D. (2008). El derecho a la ciudad. New Left Review, 53, 23-39.

Hiernaux, D., \& Lindón V. A. (2004). Desterritorialización y reterritorialización metropolitana. La Ciudad de México. Documentsd' Analisis Geogrâfica, 44, 71-88. 
Jacquin, C. (2012). Producir y habitar la periferia. Los nuevos conjuntos de vivienda de bajo costo en México. Bulletin de l'Institut Francaisd' Estudes Andines, 41(3), 389-415.

Lindón, V. A. (1997). De la expansión urbana y la periferia metropolitana. México: El Colegio de México.

Lindón, V. A. (2006).Territorialidad y género. Una aproximación desde la subjetividad espacial. En M. A. Ramírez Kuri \& P. Aguilar Díaz (Eds.), Pensar $y$ habitar la ciudad. Afectividad, memoria y significado en el espacio urbano contemporáneo (pp. 13-32). Barcelona: Anthropos.

Lucas, K. (2004).Transport and social exclusion. En K. Lucas (Ed.), Running on Empty. Transport, Social Exclusion and Environmental Justice (pp. 39-54). Bristol: Policy Press.

Manderscheid, K. (2009). Unequal Mobilities. En T. Ohnmacht, H. Maksim M. M. \& Bergman (Eds.), Mobilities and Inequality (pp. 27-50). Farnham: Ashgate.

Marcuse, P. (2003). Cities in Quarters. En G. Bridge \& S. Watson (Eds.), A companion to the city (pp. 270-281). Oxford: Blackwell.

Mason, F. P. (2007). Watching the Traffic Go by. Transportation and Isolation in Urban America. Austin: University of Texas Press.

Massey, D. (2008). Ciudad Mundial. Caracas: Fundación Editorial el Perro y la Rana.
Merton, R. K. (2007). On Sociological Theories of the Middle Range. En Calhoun, Craig (Ed.), Classical sociological theory (pp. 448-459). Oxford: Blackwell.

Mitchell, K., \& Beckett, K. (2008). Securing the Global City. Crime, Consulting, Risk and Ratings in the Production of Urban Space. Indiana Journal of Global Legal Studies, 15(1), 75-115.

Mulley, C., \& Nelson, J. (2009). Flexible Transport Services. A new Market Oportunity for Public Transport. Research in Transportation Economics, 25(1), 39-45.

Ortiz, G. A., \& Zetter, R. (2004). Market Enablement and Reconfiguration of Urban Structure in Colombia. En R. Zetter \& M. Hamza (Eds.), Market Economy and Urban Change. Impacts in the Developing World (pp. 185-204). London: Earhtscan.

Peck, J. T. N., \& Brenner, N. (2009). Neoliberal Urbanism. Models, Moments, Mutations. SAIS Review, XXXIX(1), 49-66.

Redshaw, S. (2008). In the Company of Cars. Driving as a Social and Cultural Practice. Aldershot: Ashgate.

Rodgers, D. B. J., \& Kanbur, R. (2011). Latin American Urban Development into the Twenty-first Century: Towards a Renewed Perspective of the City. European Journal of Development Research, 23(4), 550-568.

Sánchez, P. G. A. (2011). Incidencia del aeropuerto Eldorado en la estructura espacial de la ciudad de Bogotá, período 
1990-2010. Perspectiva Geográfica, 16, 173-196.

Sassen, S. (2000). Cities in a World Economy. London: Pine Forge Press.

Sibley, D. (1995). Geographies of Exclusion. Society and Difference in the West. London: Routledge.

Sohn, H. (2011). Denationalization. The Subjugation of México and its Capital City. En T. Kaminer, M. Robles Durán \& H. Sohn (Eds.), Urban Asymmetries. Studies and Projects on Neoliberal Urbanization (pp. 64-83). Rotterdam: 010 Publishers.

Soja, E. W. (1990). Postmodern Geographies. The Reassertion of Space in Critical Social Theory. London: Verso.

Soja, E. W. (2000). Postmetropolis. Critical Studies of Cities and Regions. Oxford: Blackwell.

Soja, E. W. (2003). Writing the City Spatially. City, 7(3), 269-280.
Swyngedouw, E. (2004). Globalisation or 'Glocalisation'? Networks, Territories and Rescaling. Cambridge Review of International Affairs, 17(1), 25-48.

Vázquez, C. M. T. (2004). Land privatization in México: Urbanization, Formation of Regions and Globalization in Ejidos. New York: Routledge.

Wolfe, J. (2010). Autos and Progress. The Brazilian Search for Modernity. Oxford: Oxford University Press.

Zetter, R. (2004). Market Enablement and the Urban Sector. En R. Zetter \& M. Hamza (Eds.), Market Economy and Urban Change. Impacts in the Developing World (pp. 1-40). London: Earhtscan.

Zhang, M., \& Wang, L. (2013). The impacts of mass transit on land development in China. The case of Beijing. Research in Transportation Economics, 40, 124-133. 